\title{
Reduction of Side Lobes by Using Complementary Codes for Radar Application
}

\author{
Dodda.H.V.S.Rami Reddy ${ }^{1}$, P.P.M.Krishna ${ }^{2}$,K. Ranjith Kumar ${ }^{3}$ \\ ${ }^{1}$ second. M Tech Student (DECS), ${ }^{2}$ Assoc. professor, ${ }^{3}$ Asst.professor \\ Dept of E.C.E, Dr.S.G.I.T, Markapur, Prakasam (Dist),Indial, Dept of E.C.E,S.G.I.T, Markapur ${ }^{2}$, Dept of \\ E.C.E,S.G.I.T, Markapur ${ }^{3}$ DODDA.H.V.S.Rami Reddy ${ }^{1}$, P.P.M.Krishna ${ }^{2}$,K. Ranjith Kumar ${ }^{3}$ \\ ${ }^{1}$ second. M Tech Student (DECS), ${ }^{2}$ Assoc. professor, ${ }^{3}$ Asst.professor \\ Dept of E.C.E, Dr.S.G.I.T, Markapur, Prakasam (Dist),India ${ }^{1}$, Dept of E.C.E,S.G.I.T, Markapur ${ }^{2}$, Dept of \\ E.C.E,S.G.I.T, Markapur
}

\begin{abstract}
The analysis of new types of Complementary direct sequence complex signals which have synthesized with well - know code sequences like Barker, Walsh, Golay, and complementary codes. Build on the autocorrelation function $(A C F)$ and ambiguity function $(A F)$ of signals, the numerical method estimates the volume of side lobes separately for each signal. The results obtained show that the signals, which have a low volume of side lobes, means of approximately zero in by using complementary codes with compare to different codes.
\end{abstract}

Keywords: Ambiguity function, complementary codes, autocorrelation function etc.

\section{INTRODUCTION}

In wireless communication systems such radars radio, TV and navigation systems, electromagnetic signals are transmitted in the frequency based on the different frequency bands of range from $\mathrm{KHz}$ to $\mathrm{GHz}$. In recent years many authors have published a set of research works related to estimation of ambiguity functions (AF) of these signals (communication, television or radio), i.e. estimation of the radar properties. It was concluded that the good characteristics of their ambiguity functions are not always combined with dynamic range restriction. The usage of such radars is very perspective, but related with many restrictions.

In existing system for radar communication by using the electromagnetic wave is used to find the targets in the radar receivers by extracting the received signal with the help of barker, Walsh and golay codes are used to find the location and distance of the target. But here one problem occurred i.e., high side lobes are occurred in some cases it is dominate the main lobe it causes a (False Alarm).

In proposed system to avoid the side lobes or False alarm by using the new technique i.e., complementary codes are used instead of barker, Walsh and golay codes to get almost zero side lobes easy to find the target location and distance from radar.

\section{I .Evaluation of side lobes}

In the study, we use the general definition of the following quality factors: Maximal Peak Side lobe Power (PSP), Peak Side lobe Level (PSL), Integrated Side lobe Level (ISL) and Golay Merit Factor (MF). These quality factors are often used in order to analyze the volume of signal side lobes at the filter output:

- $\quad$ Max Peak Side lobe Power $(\mathrm{PSP})=1 / \mathrm{N}$ - is the maximum amplitude at the filter output , and $\mathrm{N}$ is the code length;

- $\quad$ Peak Side lobe Level (PSL) - is the highest side lobe power level relative to the main lobe:

$$
P S L=10 \log \frac{\text { peaksidelobepower }}{\text { totalmainlobepower }} \ldots 1.1
$$

- $\quad$ Integrated Side lobe Level (ISL) - is the power distributed in all side lobes and defined as:

$$
I S L=10 \log \frac{\text { power int } \text { egratedoversidelobes }}{\text { totalmainlobepower }} \ldots 1.2
$$

- The Golay Merit Factor (MF) of binary sequences of length $\mathrm{N}$ is given by: 


$$
\begin{gathered}
M F=\frac{N^{2}}{2 \sum_{k=1}^{N-1}\left|a_{k}\right|^{2}} \ldots 1.3 \\
A A C F=a_{k}=\sum_{i=0}^{N-k-1}(-1)^{s_{i}-s_{i+k}} \quad, 1 \leq k<N
\end{gathered}
$$

$A A C F$ - Aperiodic autocorrelation function. (1.4)

\section{EXPERIMENTAL DESCRIPTION}

The study is realized by developing the specialized software in Matlab 7.6. This software computes PSL and ISL of ambiguity functions of signals under study and visualizes the results. The main quality factors of signals under study are evaluated and visualized. The structure of this software is shown in Fig. 2.1. The function of computational algorithm includes the following operations: input of the information for the type and the parameters of codes under study; calculation and normalization of the code autocorrelation function (ACF); computation of the Merit factor; calculation of PSL and ISL, graphical and numerical visualization of the final results. In case of complementary codes two codes are applied to the input; in the block for calculation of ACF a sum of two code's ACF is computed. In the next block a partial minimization of the volume of the body of ambiguity is performed resulting into a new matrix, which is normalized (a sum of matrixes in case of complementary codes). After that the quality factors PSL and ISL of the minimized ambiguity function are calculated, and, finally, the numerical and graphical results are visualized. The main purpose of this study is to analyze which of these signals have the better (lower) values of Peak Side lobe Level and Integrate Side lobe Level.

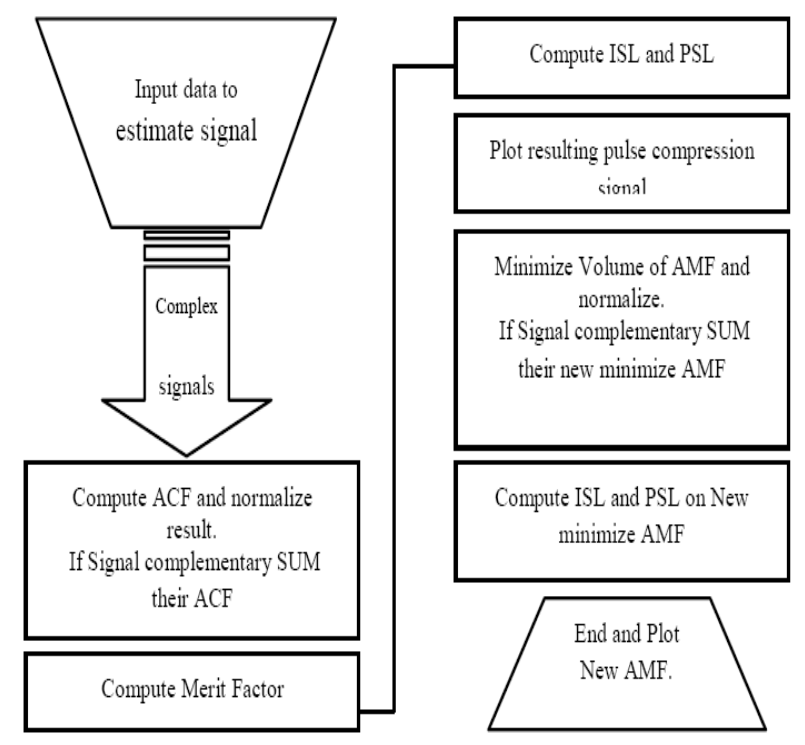

Fig 2.1: Block diagram of the computational algorithm

For the above computational algorithm is used to find the target location by applying different codes. The ex-perimental results are the given below in graphical model. 


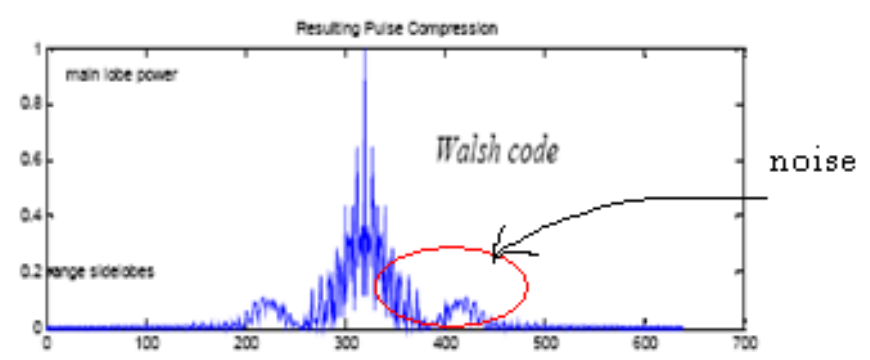

Fig 2.2.a: Autocorrelation of Walsh sequence.

In the above fig 2.2.a for detecting the target but difficult to analyze that because noise or side lobes are very high in the Walsh sequence.

The Ambiguity function for the Walsh sequence is also same but it is in 3-dimentional manner here we find easily the side lobes compare to the Autocorrelation function.

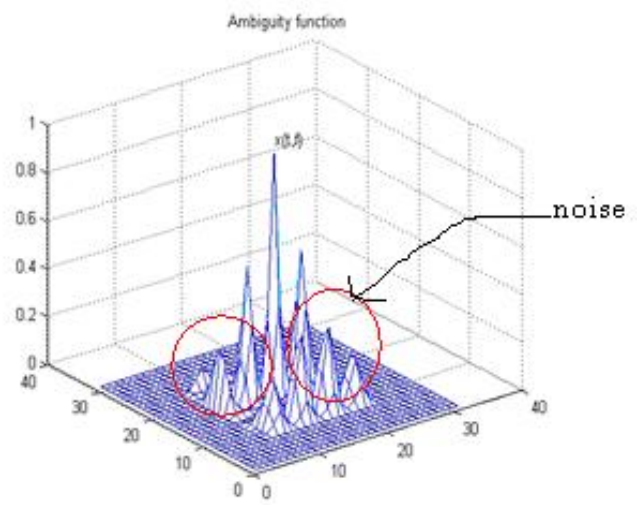

Fig 2.2.b: Ambiguity diagram of Walsh sequence.

In my proposed model by using the complementary codes to overcome the noise occurred in the Walsh codes. The results for complementary codes are given below.

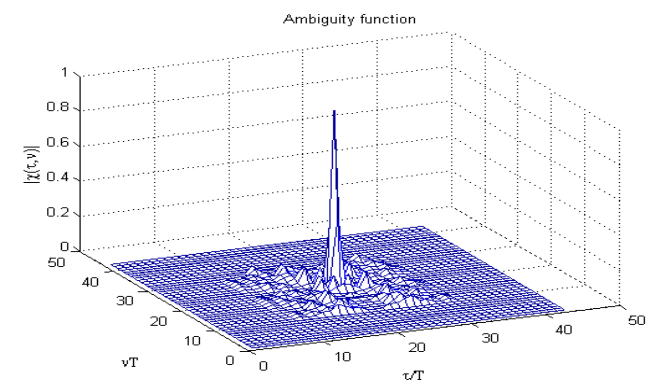

Fig 2.3.a: Autocorrelation of complementary sequence.

Compare to both autocorrelation's complementary sequence have almost zero noise. Same for ambiguity function also is in the given below. 


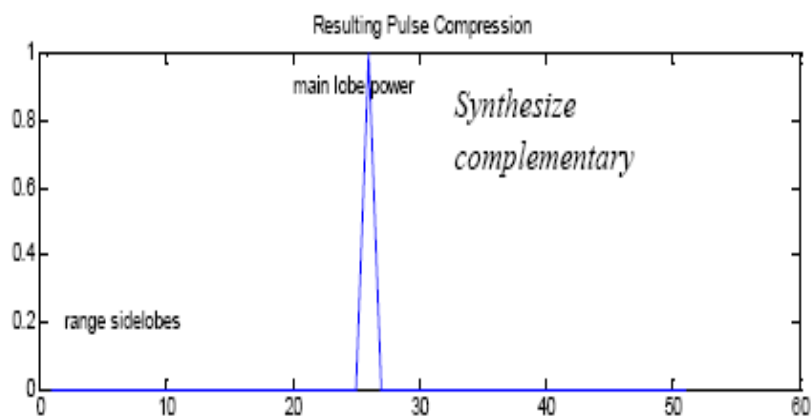

Fig.2.3.b:Ambiguity diagram of complementary sequence

\section{Conclusion}

Based on the results obtained, we conclude that the complementary codes have the best characteristics ACF and MAF in comparison with all the rest codes. The results are obtained by the software Matlab R2011a. This confirms that at the present time and in the future the complementary codes can be successfully used in passive radar systems.

\section{REFERENCES}

[1]. M. J. E. Golay, "Complementary Series, ,, IEEE Trans Info. Theory, vol. IT-7, 1961, pp 82-87.

[2]. R. Turyn, "Ambiguity Function of Complementary Sequences, ,, IEEE Trans. Info. Theory, vol. IT-9, Jan. 1963, pp. 46-47.

[3]. N. Suehiro, "Complete Complementary Code Composedof NMultipleshift Orthogonal Sequences, ,, Trans. /EICE (in Japanese), vol. J65-A, Dec. 1982, pp. 1247-53.

[4]. N. Suehiro and M. Hatori, "N-Shift Cross-OrthogonalSequences, „, IEEE Trans. Info. Theory, vol. IT-34, no. 1, Jan. 1988, pp. 14346.

[5]. Cherniakov M., Kubik M.: “Secondary applications of wireless technology (SAWT)”, 2000 European Conference on Wireless Technology - Paris 2000

[6]. Price R., E. Hofstetter, "Bounds on the Volume and Height Distributions of the Ambiguity Function", IEEE Transactions on Information Theory, April, 1965.

[7]. D.B. Vakman and R. M. Sedletskii, “Problems in Synthesis of Radar Signals”. Soviet Radio, Moscow, 1973 (in Russian).

[8]. D.E. Vakman, "Complex signals and ambiguity function” Soviet Radio, Moscow, 1965 (in Russian).

[9]. Diplom work of eng.A. Kabakchiev “Automatics synthesis of discrete complex signals". (in Bulgarian)

[10]. Dissertation of professor doctor of science, Borislav Bedzhev "Improving noise protection in radar systems on the basis of algebraic method „.. (in Bulgarian)

[11]. Hsiao-Hwa Chen, Jun-Feng Yeh, Naoki Suehiro, “A Multicarrier CDMA Architecture Based on Orthogonal Complementary Codes for New Generations of Wideband Wireless Communications"- Topics in Wireless Communication - IEEE Communication Magazine October 2001

[12]. Pingzhi Fan, Senior Member, IEEE, Weina Yuan, and Yifeng Tu" Z-complementary Binary Sequences", IEEE SIGNAL PROCESSING LETTERS, VOL. 14, NO. 8, AUGUST 2007

[13]. J. L. Walsh, “A closed set of normal orthogonal functions, , Amer. J.Math., vol. 55, pp. 5-24, 1923.

[14]. „Walsh-Like Nonlinear Phase Orthogonal Codes for Direct Sequence CDMA Communications” Ali N. Akansu, Senior Member, IEEE, and Radha Poluri

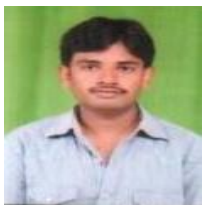

I DODDA.H.V.S.RAMI REDDY completed my B.Tech in 2010 from Loyola Institute of Technology and Management . I pursuing M.Tech in Dr Samuel George Engineering College Markapur.

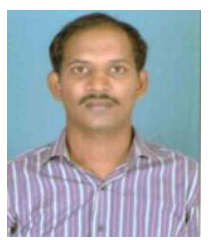

I P Prasanna Murali Krishna working as Assoc.prof in ECE Dept and also Head of the Department with Dr.SGIET Markapur from 2001 to till date.

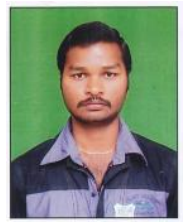

I K.Ranjith Kumar working as Asst Prof in ECE Dept with Dr.SGIET Markapur from 2012 to till date. 